\title{
Substrate Selectivity in the zDHHC Family of S-Acyltransferases
}

Kimon Lemonidis, Christine Salaun, Marianna Kouskou, Cinta Diez-Ardanuy, Luke H. Chamberlain* and Jennifer Greaves

Strathclyde Institute of Pharmacy and Biomedical Sciences, University of Strathclyde, Glasgow G4 ORE, UK.

*corresponding author: Luke.chamberlain@strath.ac.uk

Keywords: S-acylation, palmitoylation, zDHHC enzymes

\begin{abstract}
S-acylation is a reversible lipid modification occurring on cysteine residues mediated by a family of membrane-bound "zDHHC" enzymes. S-acylation predominantly results in anchoring of soluble proteins to membrane compartments or in the trafficking of membrane proteins to different compartments. Recent work has shown that although S-acylation of some proteins may involve very weak interactions with zDHHC enzymes, a pool of zDHHC enzymes exhibit strong and specific interactions with substrates, thereby recruiting them for S-acylation. For example, the ankyrin-repeat domains of zDHHC17 and zDHHC13 interact specifically with unstructured consensus sequences present in some proteins, thus contributing to substrate specificity of these enzymes. In addition to this new information on zDHHC enzyme protein substrate specificity, recent work has also identified marked differences in selectivity of zDHHC enzymes for acyl CoA substrates and has started to unravel the underlying molecular basis for this lipid selectivity. This review will focus on the protein and acyl CoA selectivity of zDHHC enzymes.
\end{abstract}

\section{Lipidation of intracellular proteins}

Intracellular proteins are subject to an array of different chemical modifications. Several of these modifications involve the attachment of lipid groups either during protein translation or following protein synthesis; lipid modifications found on intracellular proteins include $\mathrm{N}$ myristoylation, isoprenylation and S-acylation [1]. N-myristoylation is predominantly a co- 
translational modification, whereby myristate is added to a glycine at position 2 of the polypeptide chain following cleavage of the initiating methionine. In contrast, isoprenylation occurs post-translationally and involves the addition of farnesyl or geranylgeranyl groups onto C-terminal cysteines. Both $\mathrm{N}$-myristoylation and isoprenylation are irreversible and are catalysed by soluble enzymes that recognise specific consensus sequences, for example, MGXXXS/T in the case of N-myristoyltransferases and CAAX or CC in the case of isoprenyltransferases. $\mathrm{N}$-myristoylation and isoprenylation typically occur on soluble proteins and provide a means for membrane association, since these hydrophobic modifications have an affinity for the hydrophobic core of membranes. N-myristoylation and isoprenylation have been well-characterised and the enzymes that catalyse these modifications are recognised as valid drug targets, for example in cancer and infectious diseases [2, 3].

S-acylation involves the attachment of fatty acids onto cysteine residues [4]. This modification exhibits a number of important differences from $\mathrm{N}$-myristoylation and isoprenylation, including the fact that it is reversible and catalysed by polytopic membranebound enzymes [5, 6]. All classes of protein lipidation reactions have benefitted from recent technological breakthroughs, such as the use of high sensitivity click chemistry approaches with azide/alkyne fatty acid analogues; previously the study of protein lipidation relied almost exclusively on tritiated chemical probes that have a very low sensitivity of detection [7-9]. Although it is possible that the alkyne/azide groups present on the acyl probes used for click chemistry could exert an influence on the S-acylation reaction or have downstream effects on modified proteins, recent work showed that azide probes are excellent mimics of endogenous fatty acids and are converted to the expected acyl CoA species upon cell labelling [10]. Furthermore, these probes competed with endogenous fatty acids of the same chain length for conversion into acyl CoA [10]. Notwithstanding this, it is important that further general analysis of these fatty acid analogues are conducted, in particular whether the azide/alkyne groups have any influence on protein behaviour. Click chemistry has perhaps had the biggest impact on the protein S-acylation field, where prediction of substrate proteins has been more difficult than in the $\mathrm{N}$-myristoylation and isoprenylation fields, primarily due to the lack of a recognised "consensus" sequence for protein Sacylation.

Several studies have implemented click chemistry protocols together with other new approaches such as acyl-biotin exchange and acyl-RAC (resin-assisted capture) to profile the S-acylated proteome from a variety of different cell types, revealing that perhaps as much as $10 \%$ of the proteome in mammalian cells is modified by S-acylation [11-13]. Indeed, a database of all known S-acylated proteins has now been developed [14] 
(http://swisspalm.epfl.ch/), helping to establish user-friendly tools to support new investigators entering this research field.

The technical breakthroughs that have benefitted the protein S-acylation field have been complemented by important new discoveries, in particular around the enzymes that catalyse this process. Identification of the enzymes that mediate protein S-acylation lagged a long way behind similar work in the $\mathrm{N}$-myristoylation and isoprenylation fields. Moreover, enzymes that mediate protein deacylation (the reverse reaction) were identified long before the discovery of bona fide enzymes that catalyse S-acylation [15]. The big breakthrough in the search for protein acyltransferases (PATs) came through studies in Saccharomyces cerevisiae, which identified Erf2p and Akr1 as enzymes that catalyse the S-acylation of Ras and the casein kinase Yck2, respectively [16-18]. These two yeast PAT enzymes were found to share a conserved "DHHC" cysteine-rich domain (CRD), a 51 amino acid zinc finger-like domain displaying a high density of cysteine residues and with a conserved $\mathrm{DHHC}$ tetrapeptide (Akr1 actually has a DYHC sequence rather than the canonical DHHC sequence). These breakthrough discoveries served as the primer for later studies on mammalian cells that identified a family of twenty-four ZDHHC genes [6], and it is now generally accepted that $z \mathrm{DHHC}$ enzymes are responsible for the major bulk of S-acylation reactions that occur in cells [19]. However, the identification of the substrate pools of individual zDHHC enzymes has not been as successful and straightforward as may have originally been envisaged.

\section{A broad palette of proteins is regulated by S-acylation}

The range of proteins modified by S-acylation is diverse and includes both soluble and transmembrane proteins (single pass to polytopic). Soluble proteins often depend upon Sacylation to become membrane associated. In some cases, this membrane association is relatively stable and proteins do not cycle between membrane-bound and cytosolic forms [20]. However, dynamic turnover of S-acylation is an essential means to regulate membranecytosol exchange of proteins such as S-acylated Ras isoforms [21, 22] and is important in controlling the precise intracellular distribution of these proteins. Dynamic S-acylation cycles have also been reported to control membrane association of proteins such as $\mathrm{G}$ protein subunits [23] and the post-synaptic scaffold PSD95 [24]. In contrast, deacylation of the SNARE membrane fusion protein SNAP25 appears to occur at a slower rate than proteins such as Ras [25]. SNAP25 is S-acylated on up to four cysteines, and as a result of this multiple S-acylation and a slower rate of S-acylation turnover, SNAP25 is not thought to cycle between membranes and the cytosol. Instead, it was demonstrated that turnover of $S$ - 
acylation (likely on specific cysteines) acts as a means to regulate the distribution of this protein between the plasma membrane and recycling endosomes [25].

As S-acylation is mediated by membrane-associated zDHHC enzymes, modified proteins require a mechanism to associate with membranes housing the respective PAT enzyme prior to S-acylation. The role of "dual acylation" in regulating membrane association of soluble proteins is well-established [26]. Essentially, the attachment of N-myristoyl or isoprenyl chains provides a weak membrane affinity that allows the proteins to transiently associate with membranes (and hence gain access to membrane-associated zDHHC enzymes). Subsequent S-acylation of cysteines adjacent to the $\mathrm{N}$-myristoylation or isoprenylation site leads to a large increase in membrane affinity and promotes stable membrane attachment (at least of model peptides with model membranes) [26]. The precise membrane affinity of dual lipid anchors is likely to be influenced by other biochemical features of the protein but dual lipidated proteins clearly become enriched on cell membranes. As discussed above, the addition of an irreversible lipid anchor ( $N$ myristoylation or isoprenylation) and a reversible one (S-acylation) allows for dynamic regulation of membrane association via constitutive or regulated S-acylation turnover. It is not clear exactly how dynamic turnover of S-acylation on soluble proteins (or any protein) is regulated, however mechanisms regulating physical access of zDHHC enzymes to their substrates have been described [27] and there is also evidence for S-acylation enzymes being modulated by phosphorylation [28]. Similarly, phosphorylation of substrate proteins is also likely to play a role in regulating S-acylation dynamics [29, 30].

Although it is clear that S-acylation plays a major role in regulating membrane association of soluble proteins, it has been more challenging to tease apart other effects of S-acylation on these proteins. In contrast, there is an emerging wealth of information on the various effects that S-acylation has on transmembrane proteins. In particular, S-acylation appears to play an essential role in intracellular trafficking and protein stability [31, 32]. Mechanisms that have been proposed to underlie the effects of S-acylation on protein trafficking include: (i) regulating hydrophobic matching of transmembrane domains with the core of the lipid bilayer [33]; (ii) affecting the interaction of proteins with accessory trafficking factors [34]; and (iii) regulating interaction with membrane subdomains such as lipid rafts or caveolae [20,35]. Protein stability can be affected by the localisation of S-acylated proteins and there is also good evidence that blocking S-acylation can enhance ubiquitination [36], highlighting an interesting interplay between post-translational modifications in the regulation of protein stability and turnover. 


\section{S-acylation enzymes}

The identification of enzymes that mediate protein deacylation preceded the discovery of Sacylation enzymes by many years. Acyl protein thioesterase (APT) was identified as a cytosolic protein that was active against $S$-acylated substrates such as Ras proteins, $G$ alpha subunits and eNOS but which lacked activity against caveolin-1 [15, 37]. These findings are consistent with the observation that caveolin-1 S-acylation is relatively stable [38] compared with the other proteins. In addition to APT, a separate deacylation enzyme, protein palmitoyl transferase (PPT), was also identified [39, 40]. In contrast to APT, PPT is localised to lysosomes [41], where it functions in degradation of S-acylated proteins. PPT has been of additional interest due to the finding that mutations that block the activity or lysosomal targeting of this enzyme cause infantile neuronal ceroid lipofuscinosis, a rare early-onset neurodegenerative disease [42]. Recent breakthroughs have highlighted that many more thioesterase enzymes are likely to be present in the wider metabolic serine hydrolase enzyme family, and that the total number of deacylation enzymes may be on a par with the number of known PATs [43, 44].

zDHHC enzymes have now been established as the PATs that regulate the large bulk of protein S-acylation reactions. These enzymes catalyse S-acylation via a two-step reaction involving attachment of the fatty acid onto the cysteine of the DHHC motif, a process known as autoacylation, followed by transfer of the acyl group onto the substrate protein $[45,46]$. These polytopic membrane proteins are localised predominantly to endoplasmic reticulum and Golgi membranes [47, 48] but some isoforms display dynamic patterns of localisation: zDHHC2 localises to the plasma membrane and endosomes [49] and exhibits an enhanced targeting to the neuronal plasma membrane following blockade of action potential with tetrodotoxin [27]; zDHHC5 also exhibits a dynamic localisation, with neuronal activity enhancing endocytosis of this enzyme [50].

Several links have been established or suggested between zDHHC enzymes and different human disorders. A SNP in the ZDHHC8 gene has been linked to an increased susceptibility of developing schizophrenia [51], although this has not been a consistent finding across populations [52-54]. At the cellular level, knockout of zDHHC8 in mice leads to reduced dendritic complexity [55]. Mutations in zDHHC9 cause intellectual disability [56, 57] and specific disease-causing point mutations in the DHHC- cysteine-rich (CR) domain of this enzyme disrupt enzyme autoacylation [58], clearly linking defective palmitoylation to this disorder. zDHHC17 has been linked to both diabetes and Huntington's disease. zDHHC17 expression in pancreatic beta cells is reduced by inflammatory cytokines that cause beta cell dysfunction and apoptosis [59]. Indeed, RNAi-mediated depletion of zDHHC17 in a beta cell 
line led to increased apoptosis and a reduction in glucose-stimulated insulin secretion [59]. The link between zDHHC17 and Huntington's disease is based on evidence showing that this enzyme S-acylates huntingtin, which prevents the formation of inclusions [60]. Furthermore, wild-type but not mutant huntingtin was shown to positively modulate zDHHC17 enzyme activity [61]. zDHHC13 is also able to S-acylate huntingtin [61] and zDHHC13-deficient mice also develop neuropathological and behaviour feature of Huntington's disease [62]; furthermore, mutations in this enzyme can result in hair loss and osteoporosis in mice $[63,64]$. Finally, a number of zDHHC enzymes have been linked with cancer [65].

\section{Protein substrate specificity of zDHHC enzymes}

Pinning down the substrates of individual zDHHC enzymes has been more difficult than many previously envisaged. There is an ever-increasing body of information about the zDHHC enzymes that can modify specific substrate proteins, informed mainly from zDHHCsubstrate co-expression experiments. However, possible overlap in the substrate profiles of individual $z D H H C$ enzymes has limited the discovery of $z D H H C$ substrate targets through knockout/knockdown strategies. For example, proteomic analysis of the S-acylated proteome in brain samples of zDHHC17 genetrap mice identified a number of potential substrates of this enzyme but the overall reduction in the level of S-acylation of these putative substrates was quite modest [66]. In this case, this may reflect the fact that the genetrap line used has a residual level of zDHHC17 expression ( 10\%) [67] and new models with a greater ablation of $\mathrm{ZDHHC} 17$ may provide further insight into the substrates of this enzyme. An additional issue that has arisen with proteomics analyses is that changes detected in S-acylation of specific proteins sometimes actually reflect a decrease in overall protein abundance [68]. However given the known role of S-acylation in regulating protein stability [32], it is possible that proteins that exhibit changes in abundance are indeed substrates of the enzyme that has been depleted. This issue highlights the complexity of identifying zDHHC enzyme substrates, and careful follow-up analyses of proteomic datasets from $\mathrm{zDHHC}$ knockout models is essential to accurately define bona fide substrates of individual zDHHC enzymes.

zDHHC enzymes can vary substantially in their affinities for their cognate substrate proteins and in their intrinsic S-acylation activities [69]. Hence, for S-acylation of a given substrate, zDHHCs with high S-acylation activity depend more on: i) the availability of this protein in proximity to this $\mathrm{zDHHC}$ (i.e. co-localisation in the same membrane compartment), and ii) the reactivity/ neighbouring context of the cysteines to be S-acylated, rather than on a strong 
zDHHC-protein interaction; indeed the latter has been reported to be absent in the highly active zDHHC3 and zDHHC7 (Lemonidis et al 2014). On the other hand, zDHHC enzymes with low S-acylation activity are likely to exhibit stronger interactions with their respective substrates, recognizing features of the proteins that may be located far away from the Sacylated cysteines. zDHHC5 and zDHHC8 possess a long and highly disordered C-terminal tail, able to interact with structural aspects of many proteins (like PDZ domains), and such interactions quite frequently result in the S-acylation of the latter [70, 71]. zDHHC17 and zDHHC13, on the other hand, contain an ankyrin-repeat domain, which enables them to bind to a number of known zDHHC17 substrates, including huntingtin, SNAP25 and cysteine-string protein (CSP) $[69,72]$. We have recently shown that the ankyrin repeat domain of $\mathrm{zDHHC} 17$ and $\mathrm{zDHHC} 13$ can recognize disordered regions of these $\mathrm{zDHHC17}-$ substrates; this is achieved by specific recognition of an evolutionary conserved sequence in these proteins that conforms to a [VIAP][VIT]xxQP consensus [73]. This finding is similar to some other ankyrin-repeat domains that can recognise linear unstructured sequences [74, 75]. The importance of the $z \mathrm{DHHC} 17$ consensus binding site for S-acylation of the SNAP25 family of proteins had been previously demonstrated in PC12 cells, as mutation of this site led to a large decrease in membrane binding [76]. Such sequences that can serve as zDHHC17/13-binding motifs are quite abundant, with at least 20 of them present only in known zDHHC17-binding proteins [73]. Although some of these sequences may not actually interact with the ankyrin repeat domain of $\mathrm{zDHHC} 17 / 13$, it is possible to predict accurately the sequences that do bind, by establishing a sequence rule-based consensus for ankyrinrepeat binding based on peptide library screening [74]. Thus, it's possible to predict with accuracy a number of $z \mathrm{DHHC} 17 / 13$ interactors, many of which could be novel substrates of these enzymes. Indeed, current efforts in our lab are focused on this direction.

\section{Lipid substrate specificity of zDHHC enzymes}

Palmitoyl-CoA appears to be the predominant lipid substrate used in cellular S-acylation reactions, however quantification of S-acyl chains attached to cellular proteins has also revealed significant amounts of stearate and oleate attached to S-acylated proteins [77]. In addition, other work has shown that a variety of exogenously supplied fatty acids can be incorporated into cellular S-acylated proteins, including polyunsaturated fatty acids [78]. These observations are important because the type of fatty acid added to S-acylated proteins could have a major effect on its intracellular localisation and function; for example, longer chain length fatty acids might be predicted to have a greater membrane affinity than shorter fatty acids. Moreover, it is generally accepted that saturated fatty acids can target 
proteins to cholesterol-rich membrane sub-regions, whereas unsaturated fatty acids are thought to be excluded from these domains [20]. Elegant work from Jennings and Linder shed the first light on how $\mathrm{ZDHHC}$ enzymes might contribute to heterogeneity in the lipid profile of S-acylated proteins [46], by showing different fatty acyl-CoA preferences of purified zDHHC2 and zDHHC3 when the enzymes were contained in detergent micelles. The key observation made in this study was that $\mathrm{ZDHHC2}$ was able to use both $\mathrm{C} 16: 0$ and $\mathrm{C} 18: 0$ acyl CoAs equally well, whereas zDHHC3 exhibited a strong preference for C16:0 over C18:0 [46]. Recent work from our group has confirmed this observation in cells using clickable fatty acid and further shown that zDHHC enzymes more widely have marked differences in fatty acid selectivity [10]. For example, zDHHC17 and zDHHC23 exhibited a preference for C18:0 fatty acids over $\mathrm{C} 16: 0$ and $\mathrm{C} 14: 0$, whereas $\mathrm{zDHHC} 3, \mathrm{zDHHC7}$ had selectivity for shorter chain fatty acids.

zDHHC3 and zDHHC7 are closely-related enzymes that exhibit substantial overlap in their protein substrates [6]. Despite this, we observed that $\mathrm{ZDHHC7}$ used $\mathrm{C} 18: 0$ as a substrate better than zDHHC3 did [10]. To identify the molecular basis for this difference, we undertook a detailed mutational analysis of $\mathrm{zDHHC}$ and found that transmembrane domain 3 plays a key role in determining the fatty acid selectivity profile of this enzyme. Indeed, a single isoleucine residue in the middle of this transmembrane domain is critical in limiting the use of $\mathrm{C} 18: 0$ fatty acid by zDHHC3. zDHHC7 contains a serine at the same position, and an isoleucine-to-serine switch in $\mathrm{zDHHC} 3$ improved the ability of this enzyme to use C18:0 fatty acid as a substrate [10]. Transmembrane domain 3 is located immediately downstream of the catalytic DHHC-CR domain and thus the isoleucine residue in this membrane-spanning region of $z D H H C 3$ might act as a filter to determine the length of fatty acid substrate that can be added to the enzyme during 'autoacylation'.

In addition to providing new insight into mechanisms regulating fatty acid selectivity of zDHHC enzymes, this study also highlighted that azide fatty acids are very good mimics of endogenous fatty acids (as discussed above). Indeed, the fatty acid selectivity profiles that were observed for zDHHC3 and zDHHC7 using azide fatty acid probes were verified using tritiated versions of $\mathrm{C} 16: 0$ and $\mathrm{C} 18: 0$ [10].

\section{Concluding Remarks}

Mechanisms controlling the substrate specificity of zDHHC enzymes are beginning to emerge. $\mathrm{zDHHC17}$ and $\mathrm{zDHHC13}$ are key enzymes required for neuronal function and also implicated in Huntington's disease and other disorders. Our recent work shows that they 
recognise via their ankyrin-repeat a conserved consensus sequence in multiple substrates and binding partners. This new information has not only provided a mechanism to predict new targets of these enzymes and to complement proteomic analyses, but is also expected to lead to a better understanding of the disease mechanisms associated with specific enzyme dysfunction. Hence, the identification of other consensus recognition sites for zDHHC enzymes is crucial. Our recent work has shown that clickable fatty acids are excellent tools to dissect the fatty acid selectivity of $z D H H C$ enzymes and has provided a molecular basis on how this occurs for two zDHHC enzymes. It will be interesting to now investigate if similar selectivity mechanisms exist in other zDHHC enzymes, and how differences in selectivity impact endogenous protein substrates and cellular pathways.

\section{References}

1 Resh, M. D. (2006) Trafficking and signaling by fatty-acylated and prenylated proteins. Nature Chemical Biology. 2, 584-590

2 Basso, A. D., Kirschmeier, P. and Bishop, W. R. (2006) Thematic review series: Lipid Posttranslational Modifications. Farnesyl transferase inhibitors. J Lipid Res. 47, 15-31

3 Frearson, J. A., Brand, S., McElroy, S. P., Cleghorn, L. A. T., Smid, O., Stojanovski, L.,et al. (2010) $\mathrm{N}$-myristoyltransferase inhibitors as new leads to treat sleeping sickness. Nature. 464, 728-732

4 Chamberlain, L. H. and Shipston, M. J. (2015) The physiology of protein S-acylation. Physiol Rev. 95, 341-376

5 Magee, A. I., Gutierrez, L., McKay, I. A., Marshall, C. J. and Hall, A. (1987) Dynamic fatty acylation of p21N-ras. EMBO J. 6, 3353-3357

6 Fukata, M., Fukata, Y., Adesnik, H., Nicoll, R. A. and Bredt, D. S. (2004) Identification of PSD-95 Palmitoylating Enzymes. Neuron. 44, 987-996

7 Gao, X. and Hannoush, R. N. (2014) Single-cell in situ imaging of palmitoylation in fatty-acylated proteins. Nat Protoc. 9, 2607-2623

8 Yap, M. C., Kostiuk, M. A., Martin, D. D. O., Perinpanayagam, M. A., Hak, P. G., Siddam, A., et al. (2010) Rapid and selective detection of fatty acylated proteins using \{omega\}-alkynyl-fatty acids and click chemistry. J. Lipid Res. 51, 1566-1580

9 Hang, H. C., Geutjes, E.-J., Grotenbreg, G., Pollington, A. M., Bijlmakers, M. J. and Ploegh, H. L. (2007) Chemical probes for the rapid detection of fatty-acylated proteins in mammalian cells. J Am Chem Soc. 129, 2744-2745

10 Greaves, J., Munro, K. R., Davidson, S. C., Riviere, M., Wojno, J., Smith, T. K., et al. (2017) Molecular basis of fatty acid selectivity in the zDHHC family of S-acyltransferases revealed by click chemistry. Proc Natl Acad Sci U S A. 114, E1365-E1374

11 Yount, J. S., Moltedo, B., Yang, Y.-Y., Charron, G., Moran, T. M., Lopez, C. B. and Hang, H. C. (2010) Palmitoylome profiling reveals S-palmitoylation-dependent antiviral activity of IFITM3. Nat Chem Biol. 6, 610-614

12 Martin, B. R. and Cravatt, B. F. (2009) Large-scale profiling of protein palmitoylation in mammalian cells. Nat Meth. 6, 135-138

13 Kang, R., Wan, J., Arstikaitis, P., Takahashi, H., Huang, K., Bailey, A. O., et al. (2008) Neural palmitoyl-proteomics reveals dynamic synaptic palmitoylation. Nature. 456, 904-909

14 Blanc, M., David, F., Abrami, L., Migliozzi, D., Armand, F., Burgi, J. and van der Goot, F. G. (2015) SwissPalm: Protein Palmitoylation database. F1000Research. 4, 261 
15 Duncan, J. A. and Gilman, A. G. (1998) A cytoplasmic Acyl-Protein Thioesterase that removes palmitate from $G$ protein alpha subunits and p21RAS. J. Biol. Chem. 273, 15830-15837

16 Lobo, S., Greentree, W. K., Linder, M. E. and Deschenes, R. J. (2002) Identification of a Ras Palmitoyltransferase in Saccharomyces cerevisiae. J. Biol. Chem. 277, 41268-41273

17 Roth, A. F., Feng, Y., Chen, L. and Davis, N. G. (2002) The yeast DHHC cysteine-rich domain protein Akr1p is a palmitoyl transferase. J. Cell Biol. 159, 23-28

18 Bartels, D. J., Mitchell, D. A., Dong, X. and Deschenes, R. J. (1999) Erf2, a novel gene product that affects the localization and palmitoylation of Ras2 in Saccharomyces cerevisiae. Mol. Cell. Biol. 19, 6775-6787

19 Roth, A. F., Wan, J., Bailey, A. O., Sun, B., Kuchar, J. A., Green, W. N.,et al. (2006) Global analysis of protein palmitoylation in yeast. Cell. 125, 1003-1013

20 Salaun, C., Greaves, J. and Chamberlain, L. H. (2010) The intracellular dynamic of protein palmitoylation. J Cell Biol. 191, 1229-1238

21 Rocks, O., Pekker, A., Kahms, M., Verveer, P.J., Koerner, C., Lumbierres, M. et al. (2005) An acylation cycle regulates localization and activity of palmitoylated Ras isoforms. Science. 307, 1746-1752

22 Rocks, O., Gerauer, M., Vartak, N., Koch, S., Huang, Z.-P., Pechlivanis, M., et al. (2010) The Palmitoylation Machinery Is a Spatially Organizing System for Peripheral Membrane Proteins. Cell. 141, 458-471

23 Tsutsumi, R., Fukata, Y., Noritake, J., Iwanaga, T., Perez, F. and Fukata, M. (2009) Identification of $\mathrm{G}$ protein \{alpha\} subunit-palmitoylating enzyme. Mol. Cell. Biol. 29, 435-447

24 El-Husseini, A., Schnell, E., Dakoji, S., Sweeney, N., Zhou, Q., Prange, O. et al. (2002) Synaptic strength regulated by palmitate cycling on PSD-95. Cell. 108, 849-863

25 Greaves, J. and Chamberlain, L. H. (2011) Differential palmitoylation regulates intracellular patterning of SNAP25. J Cell Sci. 124, 1351-1360

26 Shahinian, S. and Silvius, J. (1995) Doubly-lipid-modified protein sequence motifs exhibit longlived anchorage to lipid bilayer membranes. Biochemistry. 34, 3813-3822

27 Noritake, J., Fukata, Y., Iwanaga, T., Hosomi, N., Tsutsumi, R., Matsuda, N., et al. (2009) Mobile DHHC palmitoylating enzyme mediates activity-sensitive synaptic targeting of PSD-95. J. Cell Biol. 186, 147-160

28 Lievens, P. M., Kuznetsova, T., Kochlamazashvili, G., Cesca, F., Gorinski, N., Galil, D. A., et al. (2016) ZDHHC3 tyrosine phosphorylation regulates neural cell adhesion molecule palmitoylation. Mol Cell Biol. 36, 2208-2225

29 Tian, L., Jeffries, O., McClafferty, H., Molyvdas, A., Rowe, I. C. M., Saleem, F., et al. (2008) Palmitoylation gates phosphorylation-dependent regulation of BK potassium channels. Proc Natl Acad Sci USA. 105, 21006-21011

30 Charych, E. I., Jiang, L.-X., Lo, F., Sullivan, K. and Brandon, N. J. (2010) Interplay of palmitoylation and phosphorylation in the trafficking and localization of phosphodiesterase 10A: Implications for the treatment of Schizophrenia. J. Neurosci. 30, 9027-9037

31 Blaskovic, S., Blanc, M. and van der Goot, F. G. (2013) What does S-palmitoylation do to membrane proteins? FEBS J. 280, 2766-2774

32 Linder, M. E. and Deschenes, R. J. (2007) Palmitoylation: policing protein stability and traffic. Nat Rev Mol Cell Biol. 8, 74-84

33 Abrami, L., Kunz, B. A., lacovache, I. and van der Goot, F. G. (2008) Palmitoylation and ubiquitination regulate exit of the Wnt signaling protein LRP6 from the endoplasmic reticulum. Proc Natl Acad Sci USA. 105, 5384-5389

34 Hayashi, T., Thomas, G. M. and Huganir, R. L. (2009) Dual Palmitoylation of NR2 Subunits Regulates NMDA Receptor Trafficking. Neuron. 64, 213-226

35 Abrami, L., Leppla, S. H. and van der Goot, F. G. (2006) Receptor palmitoylation and ubiquitination regulate anthrax toxin endocytosis. J Cell Biol. 172, 309-320 
36 Valdez-Taubas, J. and Pelham, H. (2005) Swf1-dependent palmitoylation of the SNARE Tlg1 prevents its ubiquitination and degradation. Embo J. 24, 2524-2532

37 Yeh, D. C., Duncan, J. A., Yamashita, S. and Michel, T. (1999) Depalmitoylation of Endothelial Nitric-oxide Synthase by Acyl-protein Thioesterase 1 is potentiated by Ca2+-Calmodulin. J. Biol. Chem. 274, 33148-33154

38 Parat, M.-O. and Fox, P. L. (2001) Palmitoylation of Caveolin-1 in endothelial cells Is posttranslational but irreversible. J Biol Chem. 276, 15776-15782

39 Camp, L. A. and Hofmann, S. L. (1993) Purification and properties of a palmitoyl-protein thioesterase that cleaves palmitate from H-Ras. J. Biol. Chem. 268, 22566-22574

40 Camp, L. A., Verkruyse, L. A., Afendis, S. J., Slaughter, C. A. and Hofmann, S. L. (1994) Molecular cloning and expression of palmitoyl-protein thioesterase. J. Biol. Chem. 269, 23212-23219

41 Verkruyse, L. A. and Hofmann, S. L. (1996) Lysosomal targeting of Palmitoyl-protein Thioesterase. J. Biol. Chem. 271, 15831-15836

42 Vesa, J., Hellsten, E., Verkruyse, LA., Camp, LA., Rapola, J., Santavuori, P., et al. (1995) Mutations in the palmitoyl protein thioesterase gene causing infantile neuronal ceroid lipofuscinosis. Nature. 376, 584-587.

43 Yokoi, N., Fukata, Y., Sekiya, A., Murakami, T., Kobayashi, K. and Fukata, M. (2016) Identification of PSD-95 depalmitoylating enzymes. J Neurosci. 36, 6431-6444

44 Lin, D. T. and Conibear, E. (2015) ABHD17 proteins are novel protein depalmitoylases that regulate $\mathrm{N}$-Ras palmitate turnover and subcellular localization. eLife. 4, e11306

45 Mitchell, D. A., Mitchell, G., Ling, Y., Budde, C. and Deschenes, R. J. (2010) Mutational analysis of Saccharomyces cerevisiae Erf2 reveals a two-step reaction mechanism for protein palmitoylation by DHHC enzymes. J Biol Chem. 285, 38104-38114

46 Jennings, B. C. and Linder, M. E. (2012) DHHC protein S-acyltransferases use a similar ping-pong kinetic mechanism but display different acyl-CoA specificites. J Biol Chem. 287, 7236-7245

47 Ohno, Y., Kihara, A., Sano, T. and Igarashi, Y. (2006) Intracellular localization and tissue-specific distribution of human and yeast DHHC cysteine-rich domain-containing proteins. Biochimica et Biophysica Acta (BBA) - Molecular and Cell Biology of Lipids. 1761, 474-483

48 Gorleku, O. A., Barns, A.-M., Prescott, G. R., Greaves, J. and Chamberlain, L. H. (2011) Endoplasmic Reticulum Localization of DHHC Palmitoyltransferases Mediated by Lysine-based Sorting Signals. J Biol Chem. 286, 39573-39584

49 Greaves, J., Carmichael, J. A. and Chamberlain, L. H. (2011) The palmitoyl transferase DHHC2 targets a dynamic membrane cycling pathway: regulation by a C-terminal domain. Mol. Biol. Cell. 22, 1887-1895

50 Brigidi, G. S., Santyr, B., Shimell, J., Jovellar, B. and Bamji, S. X. (2015) Activity-regulated trafficking of the palmitoyl-acyl transferase DHHC5. Nat Commun. 6, 8200

51 Mukai, J., Liu, H., Burt, R., Swor, D. E., Lai, W. S., Karayiorgou, M. and Gogos, J. A. (2004) Evidence that the gene encoding ZDHHC8 contributes to the risk of schizophrenia. Nat Genet. 36, 725-731

52 Otani, K., Ujike, H., Tanaka, Y., Morita, Y., Kishimoto, M., Morio, A., et al. (2005) The ZDHHC8 gene did not associate with bipolar disorder or schizophrenia. Neuroscience Letters. 390, 166170

53 Saito, S., Ikeda, M., Iwata, N., Suzuki, T., Kitajima, T., Yamanouchi, Y., et al. (2004) No association was found between a functional SNP in ZDHHC8 and schizophrenia in a Japanese case-control population. Neurosci Lett. 374, 21 - 24

54 Glaser, B., Schumacher, J., Williams, H. J., Jamra, R. A., lanakiev, N., Milev, R., et al. (2005) No association between the putative functional ZDHHC8 single nucleotide polymorphism rs175174 and Schizophrenia in large european samples. Biological Psychiatry. 58, 78-80

55 Mukai, J., Dhilla, A., Drew, L. J., Stark, K. L., Cao, L., MacDermott, A. B., et al. (2008) Palmitoylation-dependent neurodevelopmental deficits in a mouse model of 22q11 microdeletion. Nat Neurosci. 11, 1302-1310 
56 Masurel-Paulet, A., Kalscheuer, V. M., Lebrun, N., Hu, H., Levy, F., Thauvin-Robinet, C., et al. (2014) Expanding the clinical phenotype of patients with a ZDHHC9 mutation. Am J Med Genet. 164, 789-795

57 Raymond, F. L., Tarpey, P. S., Edkins, S., Tofts, C., O'Meara, S., Teague, J., et al. (2007) Mutations in ZDHHC9, which encodes a palmitoyltransferase of NRAS and HRAS, cause X-linked mental retardation associated with a marfanoid habitus. Am J Hum Genet. 80, 982-987

58 Mitchell, D. A., Hamel, L. D., Reddy, K. D., Farh, L., Rettew, L. M., Sanchez, P. R. and Deschenes, R. J. (2014) Mutations in the X-Linked intellectual disability gene, zDHHC9, alter autopalmitoylation activity by distinct mechanisms. J Biol Chem. 289, 18582-18592

59 Berchtold, L. A., Størling, Z. M., Ortis, F., Lage, K., Bang-Berthelsen, C., Bergholdt, R., et al. (2011) Huntingtin-interacting protein 14 is a type 1 diabetes candidate protein regulating insulin secretion and $\beta$-cell apoptosis. Proc Natl Acad Sci USA. 108, E681-E688

60 Yanai, A., Huang, K., Kang, R., Singaraja, R. R., Arstikaitis, P., Gan, L., et al. (2006) Palmitoylation of huntingtin by HIP14is essential for its trafficking and function. Nat Neurosci. 9, 824-831

61 Huang, K., Sanders, S. S., Kang, R., Carroll, J. B., Sutton, L., Wan, J., et al. (2011) Wild-type HTT modulates the enzymatic activity of the neuronal palmitoyl transferase HIP14. Hum Mol Genet. 20, 3356-3365

62 Sutton, L. M., Sanders, S. S., Butland, S. L., Singaraja, R. R., Franciosi, S., Southwell, A. L., et al. (2013) Hip14l-deficient mice develop neuropathological and behavioural features of Huntington disease. Hum Mol Genet. 22, 452-465

63 Saleem, A. N., Chen, Y.-H., Baek, H. J., Hsiao, Y.-W., Huang, H.-W., Kao, H.-J., et al. (2010) Mice with alopecia, osteoporosis, and systemic amyloidosis due to mutation in Zdhhc13, a gene coding for palmitoyl acyltransferase. PLOS Genetics. 6, e1000985

64 Song, I. W., Li, W.-R., Chen, L.-Y., Shen, L.-F., Liu, K.-M., Yen, J. J. Y., et al. (2014) Palmitoyl acyltransferase, Zdhhc13, facilitates bone mass acquisition by regulating postnatal epiphyseal development and endochondral ossification: A mouse model. PLoS ONE. 9, e92194

65 Greaves, J. and Chamberlain, L. H. (2014) New links between S-acylation and cancer. J Pathol. 233, 4-6

66 Wan, J., Savas, J.N., Roth, A.F., Sanders, S.S., Singaraja, R.R., et al. (2013) Tracking brain palmitoylation change: Predominance of glial change in a mouse model of Huntington's Disease. Chemistry \& Biology. 20, 1421-1434

67 Sanders, S. S., Parsons, M. P., Mui, K. K., Southwell, A. L., Franciosi, S., Cheung, D., et al. (2016) Sudden death due to paralysis and synaptic and behavioral deficits when Hip14/Zdhhc17 is deleted in adult mice. BMC biology. 14, 108

68 Li, Y., Martin, B. R., Cravatt, B. F. and Hofmann, S. L. (2012) DHHC5 protein palmitoylates Flotillin-2 and is rapidly degraded on induction of neuronal differentiation in cultured cells. J Biol Chem. 287, 523-530

69 Lemonidis, K., Gorleku, O. A., Sanchez-Perez, M. C., Grefen, C. and Chamberlain, L. H. (2014) The Golgi S-acylation machinery is comprised of zDHHC enzymes with major differences in substrate affinity and S-acylation activity. Mol Biol Cell. 25, 3870-3873

70 Thomas, G.M., Hayashi, T., Chiu, S.-L., Chen, C.-M. and Huganir, R.L. (2012) Palmitoylation by DHHC5/8 Targets GRIP1 to dendritic endosomes to regulate AMPA-R trafficking. Neuron. 73, 482-496

71 Howie, J., Reilly, L., Fraser, N. J., Vlachaki Walker, J. M., Wypijewski, K. J., Ashford, M. L., et al. (2014) Substrate recognition by the cell surface palmitoyl transferase DHHC5. Proc Natl Acad Sci U S A. 111, 17534-17539

72 Huang, K., Sanders, S., Singaraja, R., Orban, P., Cijsouw, T., Arstikaitis, P., et al. (2009) Neuronal palmitoyl acyl transferases exhibit distinct substrate specificity. FASEB J. 23, 2605-2615

73 Lemonidis, K., Sanchez-Perez, M. C. and Chamberlain, L. H. (2015) Identification of a novel sequence motif recognised by the ankyrin-repeat domain of zDHHC17/13 S-acyl-transferases. J Biol Chem. 290, 21939-21950 
74 Guettler, S., LaRose, J., Petsalaki, E., Gish, G., Scotter, A., Pawson, T., et al. (2011) Structural basis and sequence rules for substrate recognition by Tankyrase explain the basis for cherubism disease. Cell. 147, 1340-1354

75 Xu, C., Jin, J., Bian, C., Lam, R., Tian, R., Weist, R., et al. (2012) Sequence-specific recognition of a PxLPxI/L motif by an ankyrin repeat tumbler lock. Sci Signal. 5, ra39

76 Greaves, J., Prescott, G. R., Fukata, Y., Fukata, M., Salaun, C. and Chamberlain, L. H. (2009) The hydrophobic cysteine-rich domain of SNAP25 couples with downstream residues to mediate membrane interactions and recognition by DHHC palmitoyl transferases. Mol. Biol. Cell. 20, 1845-1854

77 Muszbek, L., Haramura, G., Cluette-Brown, J., Van Cott, E. and Laposata, M. (1999) The pool of fatty acids covalently bound to platelet proteins by thioester linkages can be altered by exogenously supplied fatty acids. Lipids. 34, S331-S337

78 Liang, X., Nazarian, A., Erdjument-Bromage, H., Bornmann, W., Tempst, P. and Resh, M. D. (2001) Heterogeneous fatty acylation of Src family kinases with polyunsaturated fatty acids regulates raft localization and signal transduction. J Biol Chem. 276, 30987-30994 